\title{
BÁM ĐIỂM PHÁT CÔNG SUÂT CỰC ĐẠI TOÀN CỤC CỦA HÊ THỐNG PIN QUANG ĐIỆN SỬ DỤNG GIẢI THUẬT DI TRUYỀn
}

\author{
TRƯOONG VIẸT ANH ${ }^{1}$, BÙI VĂN HIỀ ${ }^{1,2}$, DƯONG THANH LONG $^{3}$ \\ ${ }^{1,3}$ Khoa Điện-Điện Tử, Trường Đại học Su Phạm Kỹ Thuật Thành Phố Hồ Chí Minh \\ ${ }^{2}$ Khoa Công Nghệ Điện-Điện tử, Trường Đại học Công Nghiệp Thực Phẩm Thành Phố \\ Hồ Chi Minh \\ ${ }^{3}$ Khoa Công nghệ Điện, Trường Đại học Công nghiệp Thành Phố Hồ Chí Minh \\ duongthanhlong@iuh.edu.vn
}

Tóm tắt. Khi yêu cầu hệ thống điện với cấp điện áp và công suất lớn, thường không thể sử dụng đơn thuần cấu hình liên kết song song $(\mathrm{PC})$ vì có dòng điện ngõ ra rất lớn gây khó khăn cho việc thiết kế các mạch chuyển đổi. Thay vào đó, các cấu hình nối tiếp (SC) hoặc nối tiếp song song (SPC) được ứng dụng nhiều hơn vì dòng điện ngõ ra an toàn hơn cho các khóa điều khiển. Tuy nhiên, hai loại cấu hình này có nhược điểm là tạo nhiều điểm cực trị trong điều kiện bóng che một phần (PSC) gây khó khăn cho việc xác định chính xác điểm phát công suất cực đại toàn cục (GMPPT). Các giải pháp MPPT truyền thống chỉ hiệu quả trong môi trường vận hành đồng nhất và kém hiệu quả trong điều kiện PSC. Trong nội dung bài viết này giới thiệu giải pháp GMPPT của hệ thống pin quang điện $(\mathrm{PV})$ bằng giải thuật di truyền. Cấu hình đề xuất dùng 3 mô-đun PV loại PHM60W36 mắc nối tiếp mô phỏng trong môi trường PSIM trong mọi điều kiện vận hành. Những kết quả thu được cho thấy khi bức xạ thay đổi liên tục thì hệ thống luôn đạt hiệu suất vượt trội và tốc độ hội tụ cao.

Từ khóa: Thuật toán di truyền, Bóng che một phần, tấm pin quang điện (PV), hệ thống pin mặt trời, đặc tính P-V.

\section{GLOBAL MAXIMUM POWER POINT TRACKING OF THE PV SYSTEM USING GENETIC ALGORITHM}

\begin{abstract}
When electrical systems with large power and voltage levels are required, a parallel link configuration (PC) cannot often be used alone because the output current is so great which makes it difficult to design converter circuits. Instead, serial (SC) or parallel-serial (SPC) configurations are more applicable because the output current value safer for the control switches. However, these two configurations have the disadvantage of creating multiple extremes under partial shading (PSC), which makes it difficult to accurately determine the global maximum power generation point (GMPPT). Traditional MPPT solutions are only effective in uniform operating environments and less effective under PSC conditions. The content of this article introduces the GMPPT solution of the photovoltaic (PV) battery system using genetic algorithms. Recommended configuration uses 3 simulated series-connected PHM60W36 PV modules under PSIM environment under all operating conditions. The results obtained show that when the radiation is constantly changing, the system always achieves outstanding efficiency and high convergence speed.

Keywords: Genetic Algorithm, Partial shading, photovoltaic (PV) solar cell, solar system, P-V characteristic.

\section{GIÓ́I THIỆU}

Nhằm giảm bớt gánh nặng lên các ngành khai thác năng lượng truyền thống như hóa thạch, thủy điện...vốn gây tác động lớn đến môi trường. Các quốc gia trên thế giới đã và đang chuyển hướng sang nguồn năng lượng tái tạo bền vững, xanh, sạch, thân thiện với môi trường $[1,2,3]$. Điện mặt trời là một trong những nguồn năng lượng được ưu tiên hàng đầu cho tới thời điểm này. Tuy nhiên, nghịch lưu hòa lưới là một thách thức không nhỏ khi phải liên kết nhiều mô đun PV lại để đạt tới giới hạn của hệ thống điện $[4,5,6$, 7]. Do đó, liên kết các mô đun lại với nhau như thế nào để đạt được hiệu suất cao nhất trong tình trạng làm việc bình thường cũng như khi xảy ra sự cố cần được nghiên cứu và xem xét.
\end{abstract}


Trong nghiên cứu trước đây của chúng tôi đã chỉ ra rằng mắc song song các tấm pin quang điện sẽ cho hiệu suất cao hơn khi mắc nối tiếp trong mọi điều kiện vận hành [8]. Hơn nữa, kiểu liên kết này có số điểm cực trị ít nhất trong điều kiện bóng che một phần. Điều này khiến cho việc lựa chọn giải pháp GMPPT đơn giản hơn nhiều so với các kiểu cấu hình khác. Tuy nhiên, $\mathrm{PC}$ chỉ phù hợp với các ứng dụng yêu cầu mức điện áp và công suất thấp bởi vì điện áp ngõ ra của hệ thống các PV song song khá nhỏ gây khó khăn cho việc thiết kế các mạch chuyển đổi công suất lớn và các khóa điện phải chịu một dòng điện cao. Nó cũng là nguyên nhân gây nên tổn thất trên các mạch điều khiển.

Trong khi đó, mặc dù $\mathrm{SC}$ có nhược điểm là sinh ra nhiều cực trị trong điều kiện vận hành không đồng nhất và dễ bị sự cố đối với những mô-đun PV không được bảo vệ trong trường hợp này (hình 1$)$. Tuy nhiên nó lại cho thấy sự vượt trội về giá trị điện áp với mức dòng điện thấp, phù hợp với việc thiết kế các mạch điều khiển. Nếu giải quyết tốt vấn đề đa điểm cực trị trong các điều kiện vận hành thì đây là cấu hình phù hợp cho những yêu cầu có mức điện áp và công suất lớn [8].

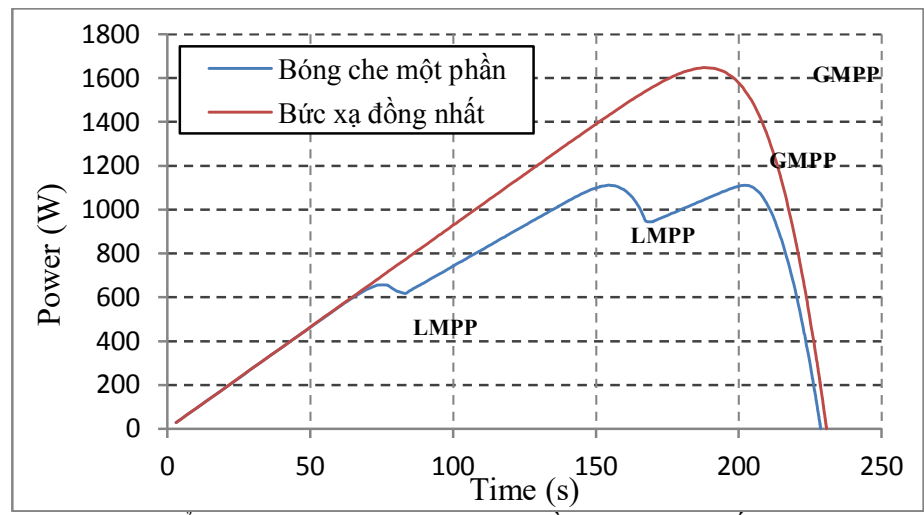

Hình 1. Ảnh hưởng bóng che một phần lên đặc tuyến của PV

Trong thực tế, để đạt giới hạn yêu cầu của hệ thống điện thường sử dụng kiểu liên kết SPC. Loại này có mức điện áp và dòng điện ở khoảng giữa so với hai cấu hình đơn lẻ nói trên nếu có cùng số lượng mô-đun PV. Tuy nhiên do cấu hình SPC có chứa liên kết SC nên nó cũng mang những nhược điểm của loại cấu hình này nên việc xuất hiện nhiều điểm cực trị cũng là điều không tránh khỏi.

Khi không thể sử dụng cấu hình $\mathrm{PC}$ cho những yêu cầu có điện áp và công suất lớn, mọi nghiên cứu chuyển sang hướng giải quyết bài toán đa điểm cực trị nhằm GMPPT và tránh LMPP trong mọi điều kiện vận hành. Để giải quyết vấn đề này, các thuật toán MPPT từ truyền thống đến tối ưu lần lược được giới thiệu. Trong đó, các nghiên cứu trước đây đã chỉ ra rằng; nhóm giải thuật truyền thống được biết đến với tên gọi thuật toán leo đồi có chung những đặc điểm như: đơn giản, dễ thực hiện, tốc độ hội tụ khá cao. Nhưng ngược lại nó có hiệu suất và độ chính xác thấp, chỉ phù hợp với môi trường vận hành đồng nhất, dễ bị bẫy vào LMPP trong điều kiện bóng che một phần [9]. Trong khi đó, nhóm thuật toán tối ưu được giới thiệu sau này chủ yếu mô phỏng những hành vi chọn lọc trong tự nhiên cho thấy khả năng đáp ứng tốt hơn. Chúng thể hiện khả năng vượt trội khi tránh được các LMPP để xác định chính xác GMPP trong mọi điều kiện vận hành như: tối ưu hóa bầy đàn (PSO) [10,11], tối ưu thuộc địa đàn kiến (ACO) [12], tối ưu bầy sói xám [13]. Tuy nhiên, mỗi giải pháp đưa ra đều có những ưu điểm, nhược điểm riêng bao gồm: Để tăng tốc độ hội tụ các giải pháp thường chọn lựa bước lặp lớn nên hiệu suất MPPT khá thấp khiến cho việc khai thác công suất của hệ thống bị hạn chế. Ngược lại, với bước lặp nhỏ sẽ gia tăng hiệu suất nhưng lại khiến cho tốc độ hội tụ tăng lên đáng kể.

Tốc độ hội tụ, hiệu suất MPPT, sự phù hợp, chi phí hợp lý, hoạt động ổn định... là những chỉ tiêu đánh giá tính hiệu quả của một giải pháp ứng với mỗi cấu hình liên kết. Rất khó để thực hiện một thí nghiệm thực tế với số lượng lớn PV trong điều kiện bóng che một phần. Bài viết này giới thiệu thuật toán di truyền (GA) áp dụng trong một cấu hình SC gồm 3 mô-đun PV loại PHM60W36 dưới sự hỗ trợ của phần mềm PSIM mô phỏng tác động của bóng che một phần để xác định GMPP của hệ thống. Những kết quả mô phỏng đạt được trên cấu hình máy tính với bộ xử lý Intel(R) Core(TM) 2 Duo CPU T5800 @ 2.00GHz, RAM 3.00GB. Cấu trúc và nguyên lý hoạt động của giải pháp đề xuất được giới thiệu trong phần 2 . Trong khi đó, những 
kết quả mô phỏng và thảo luận được trình bày trong phần 3 . Và cuối cùng, những kết luận của bài viết được trình bày trong phần 4 .

\section{2. ĐẶC TÍNH PIN QUANG ĐIỆN VÀ CẤU HÌNH HỆ THỐNG}

\subsection{Cấu hình hệ thống $P V$ đề xuất.}

Với mỗi tế bào quang điện có cấu trúc như hình 2 thì quan hệ dòng điện và điện áp ra của nó được trình bày theo biểu thức 1

$$
I=I_{S C}-I_{0}\left\{e^{\frac{q\left(V+I . R_{S}\right)}{k T}}-1\right\}-\frac{V+I_{R} R_{S}}{R_{P}}
$$

Trong đó:

$\mathrm{I}_{0}$ - dòng điện bão hòa của diode $(\mathrm{A})$;

$\mathrm{I}_{\mathrm{SC}}$ - dòng điện ngắn mạch $(\mathrm{A})$

q - điện tích của electron $\left(1,602.10^{-19} \mathrm{C}\right)$;

$\mathrm{k}$ - hằng số Boltzman $\left(1,381.10^{-23} \mathrm{~J} / \mathrm{K}\right)$;

$\mathrm{T}$ - nhiệt độ lớp tiếp xúc $(\mathrm{K})$;

$\mathrm{V}$ - điện áp ra của $\mathrm{PV}(\mathrm{V})$;

I - dòng điện ra của PV (A).

$\mathrm{R}_{\mathrm{S}}, \mathrm{R}_{\mathrm{P}}$ : điện trở nối tiếp và song song $(\Omega)$

Trong nội dung bài viết này sử dụng cấu hình $\mathrm{SC}$ nên dòng điện ra của hệ thống là không đổi và tương đương với dòng qua mỗi PV. Thông số PV được sử dụng trong nghiên cứu này được liệt kê trong bảng 1 .

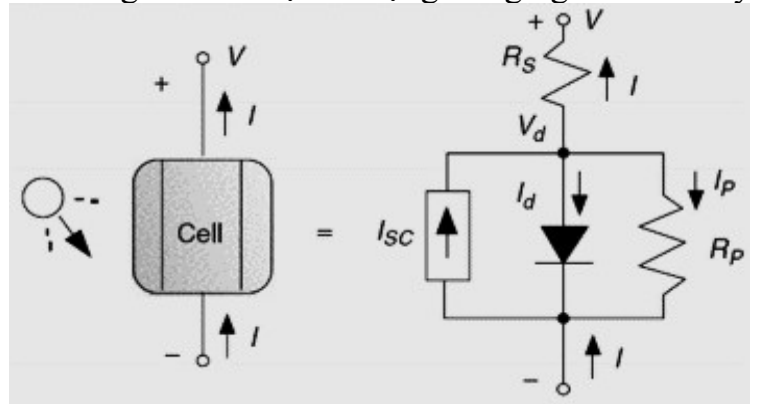

Hình 2. Sơ đồ tương đương tế bào pin quang điện

Cấu hình hệ thống PV đề xuất gồm ba mô-đun PV loại PHM60W36 mắc nối tiếp như hình 3. Một bộ MPPT để xác định giá trị độ rộng xung nhằm điều khiển bộ biến đổi $\mathrm{DC}-\mathrm{DC}$ sao cho giá trị điện áp đặt lên tải $\mathrm{R}$ thay đổi, từ đó thay đổi công suất tiêu thụ phù hợp với công suất cực đại của hệ thống PV. Hệ thống được mô phỏng trong cùng điều kiện nhiệt độ tiêu chuẩn $25^{\circ} \mathrm{C}$ nhưng với những bức xạ bề mặt khác nhau. Ngoài $\mathrm{ra}$, việc thay đổi bức xạ trên hệ thống liên tục cũng được xem xét mô phỏng trong cấu hình đề xuất đánh giá chính xác khả năng GMPPT trong những môi trường thay đổi liên tục.

Bảng 1. Thông số của một mô-đun PV

\begin{tabular}{|l|c|}
\hline \multicolumn{1}{|c|}{ Tên module PV } & PHM60W36 \\
\hline Số lượng cell & 36 \\
\hline Công suất cực đại & $60 \mathrm{~W}$ \\
\hline Dòng điện tại Pmax & $3.33 \mathrm{~A}$ \\
\hline Điện áp tại Pmax & $18 \mathrm{~V}$ \\
\hline Dòng điện nắn mạch Isc & $3.99 \mathrm{~A}$ \\
\hline Điện áp hở mạch Voc & $21.6 \mathrm{~V}$ \\
\hline Kích thước & $780 \times 675 \times 35 \mathrm{~mm}$ \\
\hline
\end{tabular}




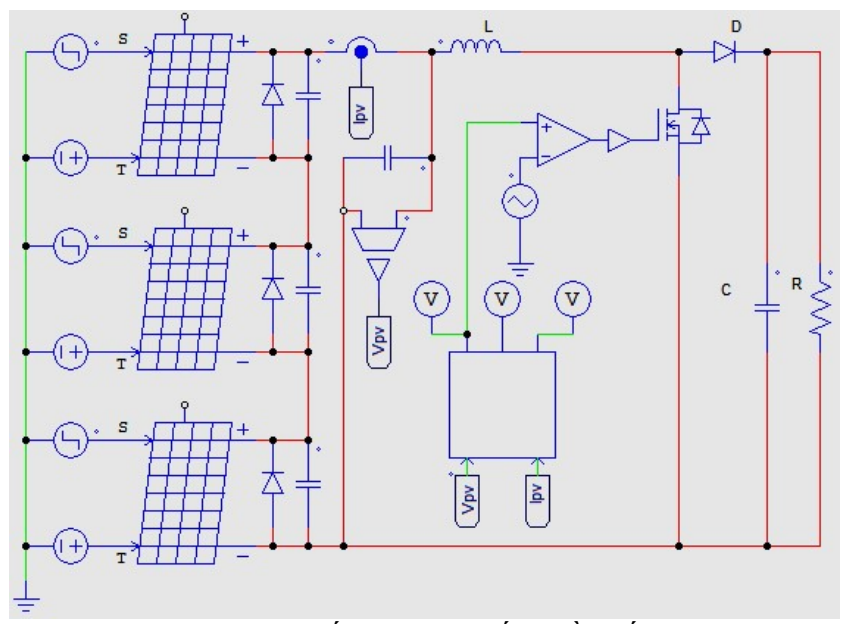

Hình 3. Cấu hình hệ thống đề xuất

\subsection{Giải thuật dò tìm MPPT.}

Để giải quyết bài toán đề ra, nội dung bài viết sử dụng giải thuật GA là kỹ thuật phỏng theo quá trình thích nghi tiến hóa của các quần thể sinh học dựa trên học thuyết Darwin. GA là phương pháp tìm kiếm tối ưu ngẫu nhiên bằng cách mô phỏng theo sự tiến hóa của con người hay của sinh vật. Ý tưởng của GA là mô phỏng các hiện tượng tự nhiên, là kế thừa và đấu tranh sinh tồn. So với các thuật toán tối ưu khác, GA có những ưu điểm nổi bật nên được đề xuất sử dụng trong nghiên cứu này như:

- GA làm việc với bộ mã của biến chứ không phải làm việc trực tiếp trên biến.

- Hầu hết các kĩ thuật tối ưu thông thường tìm kiếm từ một đỉnh, trong khi đó GA luôn hoạt động trên tập hợp đỉnh (điểm tối ưu), điều này là một ưu điểm của GA giúptăng cơ hội tiếp cận tối ưu toàn cục và tránh hội tụ sớm tại điểm cực trị địa phương.

- GA đánh giá hàm mục tiêu để phục vụ quá trình tìm kiếm, vì vậy có thể ứng dụng cho bất kì bài toán tối ưu nào (liên tục hay rời rạc).

- GA thuộc lớp các thuật toán xác suất, các thao tác cơ bản của GA dựa trên khả năng tích hợp ngẫu nhiên trong quá trình xử lý.

- Hoạt động của GA đơn giản là việc mô phỏng sự tiến hóa và chọn lọc tự nhiên bằng việc bắt đầu từ một quần thể ngẫu nhiên. Bên cạnh đó để tối ưu ta cần so sánh tuyển chọn để chọn cá thể tốt và loại bỏ cá thể xấu.

Lưu đồ giải thuật của giải pháp đề xuất được trình bày trong hình 4 .

Từ lưu đồ giải thuật có thể thấy giải pháp đề xuất hoạt động theo các bước cơ bản như sau:

Bước 1. Tạo quần thể ban đầu:

Các phần tử ban đầu của quần thể được tạo ngẫu nhiên phục vụ cho việc khảo sát công suất ra của PV khi có bóng che. Phạm vi khảo sát MPPT sẽ bị giới hạn từ $0 \div 0.8 \mathrm{R}$ là giá trị quần thể các phần tử có chứa giá trị độ rộng xung D điều khiển mạch DC-DC. Trong đó R là giới hạn độ rộng xung cực đại. Quy trình này hoạt động khi khởi động hoặc có sự thay đổi bức xạ mặt trời làm đặc tuyến đường cong $\mathrm{P}-\mathrm{V}$ thay đổi.

Bước 2. Xử lý mẫu:

Ứng với mỗi giá trị $\mathrm{D}$ được cấp trong bước 1 sẽ tiến hành đo lấy mẫu các thông số dòng điện, điện áp ngõ ra của hệ thống để xác định công suất tương ứng. Các thông số khảo sát này sẽ được chọn lọc, sắp xếp và lưu trữ cho quá trình lai tạo và đột biến kế tiếp.

Bước 3. Lai tạo và đột biến:

Trong quá trình lao tạo giữa hai cá thể tối ưu của bước 2 để tạo ra quần thể mới luôn bảo đảm giới hạn hoạt động của bộ chuyển đổi DC-DC nghĩa là giá trị độ rộng xung trong khoảng $0.8 *$ R. Các hệ số cố định và ngẫu nhiên được sử dụng trong quá trình lại tạo để tạo ra sự đột biến về giá trị trong thế hệ con.

Bước 4. Xác định hội tụ:

Quá trình hội tụ được xác định khi thỏa mãn sai số về công suất và độ rộng xung giữa hai bước lặp liên tiếp theo điều kiện:

- Điểm cho công suất đầu ra tốt nhất sau 4 thế hệ lai tạo và đột biến là MPPT

- Ba điểm tốt nhất có giá trị gần bằng nhau từ thế hệ lai thứ 2 trở đi thì điểm đó là MPPT 




Hình 4. Lưu đồi giải thuật GA đều xuất

\section{KẾT QUẢ MÔ PHỎNG}

\subsection{Các trường hợp mô phỏng thử nghiệm.}

Từ cấu hình đề xuất và các trường hợp thử nghiệm bóng che một phần cho trong bảng 2 với công suất tổng $\mathrm{P}_{\max }$ là tổng công suất thực của hệ thống được phát bởi các mô-đun riêng biệt ứng với các giá trị bức xạ khác nhau. Các trường hợp đề xuất thử nghiệm cho thấy giá trị GMPP có thể là điểm đầu (TH8), điểm giữa (TH1, TH2, TH7) hay điểm cuối (TH9) như trong hình 5. Điều đó thể hiện sự đa dạng, phong phú trong việc lấy mẫu và tính khách quan của giải pháp đề xuất khi giá trị điểm cần tìm không tập trung tại một vùng cố định. Riêng TH10 là trường hợp đặc biệt khi bức xạ trên toàn hệ thống là đồng nhất nên đường cong công suất chỉ có một cực trị.

Ngoài việc mô phỏng giải thuật GA với cấu hình đề xuất trong điều kiện bóng che một phần cố định, các trường hợp bức xạ thay đổi liên tục giữa các trường hợp cũng được xem xét vận hành một cách độc lập để kiểm tra tính hiệu quả của giải pháp đề xuất. Hơn nữa, để làm nổi bật hơn nữa ưu điểm của thuật toán, hệ thống cũng được mô phỏng song song với giải thuật PSO trong cùng các điều kiện vận hành. Chi tiết về giải thuật PSO có thể được tìm thấy trong [10]. 




Hình 5. Công suất đỉnh của một số trường hợp đề xuất.

Bảng 2. Các trường hợp mô phỏng GMPPT hệ thống

\begin{tabular}{|l|l|c|c|c|c|}
\hline Stt & $\begin{array}{c}\text { Bức xạ trên } \\
\mathbf{P V}_{\mathbf{1}} \mathbf{P V}_{\mathbf{2}-\mathbf{P V}_{\mathbf{3}}}\end{array}$ & $\begin{array}{c}\text { Công suất tổng } \\
\mathbf{P}_{\max }(\mathbf{W})\end{array}$ & $\begin{array}{c}\text { Công suất } \\
\text { MPPT (W) }\end{array}$ & $\begin{array}{c}\text { Hiệu suất } \\
\text { MPPT }\end{array}$ & $\begin{array}{c}\text { Tốc độ } \\
\text { hội tụ (s) }\end{array}$ \\
\hline 1 & $800-900-1000$ & 154.77 & 154.57 & 99.87 & 0.136 \\
\hline 2 & $1000-500-200$ & 65.82 & 65.77 & 99.92 & 0.139 \\
\hline 3 & $900-600-200$ & 77.77 & 77.32 & 99.42 & 0.141 \\
\hline 4 & $1000-750-300$ & 96.61 & 90.01 & 93.17 & 0.137 \\
\hline 5 & $800-500-200$ & 64.82 & 64.70 & 99.81 & 0.135 \\
\hline 6 & $200-500-750$ & 64.48 & 64.35 & 99.80 & 0.134 \\
\hline 7 & $700-1000-300$ & 90.72 & 87.92 & 96.91 & 0.138 \\
\hline 8 & $200-300-700$ & 42.14 & 38.89 & 92.29 & 0.135 \\
\hline 9 & $600-300-400$ & 59.01 & 58.96 & 99.91 & 0.137 \\
\hline 10 & $1000-1000-1000$ & 181.53 & 180.07 & 99.19 & 0.133 \\
\hline
\end{tabular}

\subsection{Kết quả mô phỏng GMPPT và thảo luận.}

Với giải thuật GA đề xuất khi mô phỏng trong môi trường PSIM cho thấy tất cả các trường hợp đều xác định chính xác GMPPT. Dạng sóng ngõ ra của hệ thống qua tất cả các trường hợp được trình bày trong hình 6. Ngoài ra, thông số trong bảng 2 cũng chỉ ra rằng. hiệu suất và tốc độ của giải pháp cũng là một lợi thế khi luôn đạt hơn $90 \%$ với thời gian ít hơn $0.15 \mathrm{~s}$. Điểu đó cho thấy cho dù vận hành ở môi trường đồng nhất (TH10) hay không đồng nhất (các trường hợp còn lại) thì hệ thống luôn tránh được bẫy LMPP để đạt được giá trị tốt nhất. Khi có độ chênh lệch lớn về bức xạ mặt trời khiến cho đỉnh cực trị toàn cục lệch về phía đầu hoặc cuối cùng của đường cong đặc tuyến thì hiệu suất của hệ thống khá thấp $(92.29 \%$ - TH8). Điều này được hiểu là do giới hạn của mạch chuyển đổi $\mathrm{DC}-\mathrm{DC}$ hoạt động hiệu quả trong khoảng $0.2 \mathrm{D} \div 0.8 \mathrm{D}$ độ rộng xung.

Ngoài các trường hợp mô phỏng bóng che cố định như trên, khi cho hệ thống hoạt động trong điều kiện bóng che thay đổi liên tục, kết quả thu được có dạng như hình 7 . Trong hình này, hệ thống được giả định thay đổi từ TH2 lên TH1. Kết quả cho thấy; khi có sự thay đổi bức xạ, hệ thống luôn tự điều chỉnh để bám điểm GMPP một cách chính xác. 




Hình 6. Dạng sóng GMPPT của các trường hợp thí nghiệm

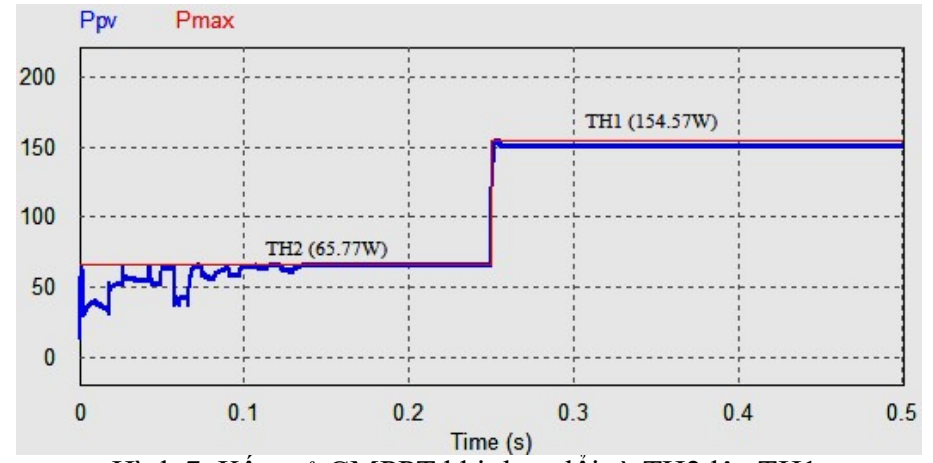

Hình 7. Kết quả GMPPT khi thay đổi từ TH2 lên TH1.

Không những thế, khi bức xạ có sự thay đổi từ mức cao xuống mức thấp (hình 8 ) thì cấu hình đề xuất luôn chứng tỏ tính hiệu quả khi bám điểm GMPPT với hiệu suất cao và tốc độ đáng kể.



Hình 8. Kết quả GMPPT khi thay đổi từ TH10 xuống TH7

Trong khi đó, trong các hình 9 và 10 thể hiện sự so sánh về tính hiệu quả của giải thuật GA với PSO. Trong hầu hết các trường hợp mô phỏng, đặc biệt là trong các tình huống bóng che một phần cố định, cả hai giải thuật đều có hiệu suất và tốc độ hội tụ tương đồng như nhau. Nhưng khi môi trường vận hành thay đổi thì một số trường hợp có các đỉnh cực trị xấp xỉ nhau khiến cho giải thuật PSO bị bẫy vào LMPP (hình 10 ). Trong hình này đã cho thấy, mặc dù tại xuất phát điểm cả hai đường Pga và Ppso đều tiệm cận giá trị Pmax. Tuy nhiên do không có khả năng đột biến như trong GA nên khi hai đỉnh cực trị gần nhau thì giải thuật PSO xác định nhầm giá trị GMPPT. Nhờ có khả năng kế thừa (lai tạo) và sự đột biến mà GA đã chứng tỏ sự vượt trội hơn trong trường hợp này. Cũng nhờ khả năng này mà trong hai hình 9 và 10 cũng đã chỉ ra rằng, tốc độ tiệm cận điểm cực trị của GA là nhanh hơn hẳn so với PSO và với độ dao động ít hơn hẳn. Điều này cho thấy ngoài việc có hiệu suất và tốc độ cao, giải pháp đề xuất cũng chứng tỏ khả năng ổn định các giá trị ngõ ra một cách thuyết phục. 




Hình 9. So sánh kêt quả MPPT bằng GA và PSO từ TH10 xuống TH7

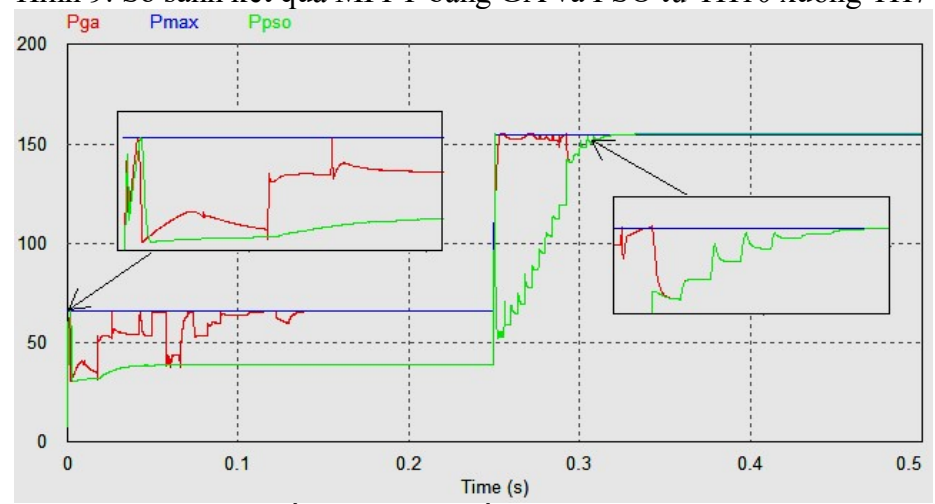

Hình 10. So sánh kết quả MPPT bằng GA và $\mathrm{PSO}$ từ TH2 lên TH1

Từ những kết quả mô phỏng trình bày trong các hình 6 đến hình 10 cùng với bảng giá trị hiệu suất và tốc độ hội tụ (bảng 2) cho thấy:

- Giải thuật GA đề xuất khá phù hợp với cấu hình trong việc GMPPT và tránh bẫy LMPP trong mọi điều kiện vận hành.

- Mặc dù GMPPT ở bất kỳ vị trí nào trên đường cong đặc tuyến công suất thì giải pháp đề xuất luôn tỏ ra hiệu quả khi xác định chính xác với hiệu suất đáng kể và tốc độ cao.

- Không những trong môi trường đồng nhất, bóng che cố định mà ngay cả khi bức xạ trên hệ thống thay đổi liên tục theo chiều hướng tăng (hình 7) hoặc giảm (hình 8) thì giải pháp luôn chứng tỏ ưu thế vượt trội khi bám điểm cực trị một cách chính xác.

- Ngoài hiệu suất cao, tốc độ GMPPT nhanh, giải thuật GA còn cho thấy khả năng ổn định các giá trị ngõ ra đáp ứng được nhu cầu ứng dụng thực tế trong các điều kiện vận hành.

\section{KẾT LUẬ̂}

Bài viết giới thiệu một giải pháp GMPPT hệ thống PV trong mọi điều kiện vận hành bằng cách sử dụng giải thuật GA. Những kết quả mô phỏng hệ thống cho thấy rằng: đối với những hệ thống không thể sử dụng cấu hình PC để tận dụng ưu điểm của nó thì giải quyết bài toán đa điểm cực trị chính là lựa chọn tối ưu. Như trình bày trong những phần trước, các giải thuật tối ưu hóa có lợi thế hơn các giải pháp truyền thống khi tránh được bẫy LMPP để đạt được hiệu quả cao với tốc độ hội tụ đáng kể. Với hiệu suât của hệ thống thấp nhất là $92.29 \%$ và tốc độ hội tụ tối đa là $0.141 \mathrm{~s}$, giải pháp đề xuất trong nội dung bài viết này đã chứng tỏ tính hiệu quả và khả năng ứng dụng của nó vào những hệ thống có yêu cầu mức điện áp và công suất lớn. Ngoài ra, khả năng đáp ứng nhanh chóng với độ dao động ngõ ra ít trong khi điều kiện môi trường thay đổi liên tục cũng là một trong những ưu điểm nổi bật mang tính ứng dụng cao của giải pháp.

\section{TÀI LIỆU THAM KHẢO}

[1] Kawamura H, Naka K, Yonekura N, Yamanaka S, Kawamura H, Ohno H (2003), Simulation of I-V characteristics of a PV module with shaded PV cells. Solar Energy Materials \& Solar Cells. 
[2] Gow JA, Manning CD (1999). Development of a photovoltaic array model for use in power-electronics simulation studies. IEE Proceedings-Electric Power Appli-cations

[3] Markvart T (2000).. Solar electricity 2nd ed. New York: John Wiley \& Sons.

[4] Wang YJ, Pierrat L (2006). Measurement of photovoltaic cell parameters for EMTP simulation. In: Proceedings of the 3rd European PV-hybrid and mini-grid conference.

[5] Gautam NK, Kaushika ND (2001). Network analysis of fault-tolerant solar photovol-taic arrays. Solar Energy Materials \& Solar Cells

[6] Wang YJ, Hsu PC (2008). Development of a piecewise linear parallel branches model for solar cell module. In: International conference on energy security and climate change. Bangkok, Thailand.

[7] R. Ramaprabha and B. L. Mathur (2012). A Comprehensive Review and Analysis of Solar Photovoltaic Array Configurations under Partial Shaded Conditions, Hindawi Publishing Corporation International Journal of Photoenergy.

[8] Trương Việt Anh, Bùi Văn Hiền, Dương Thanh Long, Lương Xuân Trường (2020). Đánh giá tác động của hiện tượng bóng che lên đặc tính làm việc của các cấu hình kết nối pin mặt trời, Tạp chi Khoa học và Công nghệ - Truờng Đại Học Công Nghiệp Thành Phố Hồ Chi Minh.

[9]. Bùi Văn Hiền, Trương Việt Anh, Quách Thanh Hải (2020). Tối ưu điểm phát công suất cực đại của pin quang điện làm việc trong điều kiện bóng che, Tạp chí Phát triển Khoa học và Công nghệ -Kĩ thuật và Công nghệ, 3(1):326338

[10]. Chun-Liang Liu et al (2012) A PSO-based MPPT Algorithm for Photovoltaic Systems Subject to Inhomogeneous Insolation, SCIS-ISIS 2012, Kobe, Japan, November 20-24.

[11]. Muhannad Alshareef, Zhengyu Lin, Mingyao Ma and Wenping Cao (2019). Accelerated Particle Swarm Optimization for Photovoltaic Maximum Power Point Tracking under Partial Shading Conditions, Energies

[12]. Titri S, Larbes C, Toumi KY, Benatchba K (2017). A new MPPT controller based on the ant colony optimization algorithm for photovoltaic systems under partial shading conditions. Appl Soft Comput; 58: 465-79

[13]. Mohanty S, Subudhi B, Ray PK (2016). A new MPPT design using Grey Wolf optimization technique for photovoltaic system under partial shading conditions. IEEE Trans Sustain Energy; 7(1):181-8.

Ngày nhận bài: 08/03/2021

Ngày chấp nhận đăng: 31/05/2021

C 2021 Trường Đại học Công nghiệp thành phố Hồ Chí Minh 